ventricular arrhythmias and 1 was found to have incidental abnormal myocardial uptake on PET imaging. 8 of the 9 patients had abnormal myocardial uptake on PET imaging. All but 1 patient had been initially treated with oral steroids ( 1 refused) and 7 of the 9 patients had also been given oral DMARDs; methotrexate $(n=6)$, azathioprine $(n=2)$, hydroxychloroquine $(n=2)$ and mycophenolate mofetil $(n=1)$. Biologics used were adalimumab $(n=5)$, infliximab $(n=3)$ and rituximab $(n=1)$. The most common indication for biologics was progression of disease despite optimal doses of standard therapy, followed by intolerance or contraindication to standard therapy. $75 \%$ of the patients were noted to have marked clinical improvement with the addition of a biologic. 4 out of 9 patients had decreased myocardial uptake on PET following treatment with a biologic. One patient had no change on PET and 4 have not had repeat imaging done yet. None of the patients had worsening of left ventricular systolic function with the addition of a TNF alpha antagonist. There were no reported major infections or significant adverse events that were attributable to the use of biologics.

Conclusion: Based on our small cohort, biologics (mainly TNF alpha antagonists) appear to be safe and efficacious as salvage therapy for cardiac sarcoidosis. However, there is a need for prospective studies to further validate these findings as well as to identify the subset of patients that would benefit from early initiation of these therapies.

\section{REFERENCES:}

[1] Birnie, D. H., Nery, P. B., Ha, A. C., \& Beanlands, R. S. B. (2016). Cardiac Sarcoidosis. Journal of the American College of Cardiology, 68(4), 411.

Disclosure of Interests: None declared DOI: 10.1136/annrheumdis-2019-eular.5193

\section{FRI0610 CRYOPYRIN-ASSOCIATED PERIODIC SYNDROME: A SERIE OF CASES IN A ADULT'S PATIENTS COHORT WITH AUTOINFLAMMATORY SYNDROME IN A THIRD LEVEL HOSPITAL}

Alberto Ruiz Román ${ }^{1}$, Salvador García Morillo ${ }^{1}$, Marco Montes Cano², Clara Aguilera Cros ${ }^{1}$, Esteban Rubio Romero ${ }^{1}$, Ricardo Gil Velez ${ }^{1} .{ }^{1}$ Hospital Virgen del Rocio, Rheumatology, Seville, Spain; ${ }^{2}$ Hospital Virgen del ROcio, Immunology, Seville, Spain

Background: The Cryopyrin-associated Periodic Syndrome (CAPS) or cryopyrinopathies are part of the spectrum of autoinflammatory diseases. The term cryopyrinopathy encompasses three clinical entities such as: CINCA/NOMID (chronic infantile neurologic, cutaneous and articular/neonatal onset multisystem inflammatory disease), Muckle-Wells disease and FCAS (familial cold-associated periodic syndrome). There are three different diseases that would be part of the same clinical spectrum, ranging from CINCA/NOMID (more serious) to FCAS (the less severe). It is more frequent in pediatric age although they can also be observed in adulthood. They are characterized by mutations in the NLRP3 gene and have an autosomal dominant inheritance pattern

Objectives: To describe the clinical characteristics and genetic variants of a cohort of patients in adulthood diagnosed with cryopyrinopathy and with follow-up in a 3rd level hospital

Methods: Retrospective descriptive study in adults patients with diagnosis of cryopyrinopathy since 2013 (year of introduction of genetic tests in the hospital laboratory) until now. The data was obtained from the review of medical records. All patients with mutations in NLRP3 gene and clinically compatible with this diagnosis were reviewed.

Results: Of a total of 44 patients in adulthood diagnosed with periodic fever syndromes (FMF, TRAPS, cryopyrinopathies, HIDS) and compatible genetic mutations, 7 patients $(15.9 \%)$ were diagnosed with cryopyrinopathies, presenting 6 of them $(13,6 \%$ ) mutations in NLRP3 gene. 1 patient was diagnosed with Muckle Wells based on clinical criteria without genetic test. 6 patients $(13.6 \%)$ were women. 4 patients $(9.1 \%)$ presented mutations in heterozygosis in exon 3 of NLRP3 gene (p.V198M). 1 patient presented heterozygous mutation in exon 3 of NLRP3 gene (p.R260W) and another patient presented a mutation in heterozygosis in exon 3 of the NLRP3 gene (p.S726G). The mean age at diagnosis was 38 years (IR 13-71 years). The totality of patients diagnosed with cryopyrinopathy showed elevation of acute phase reactants. 6 patients $(13.6 \%)$ presented fever, joint symptoms (arthralgias and/or arthritis) and myalgias. 4 patients $(9.09 \%)$ showed cutaneous involvement in the form of urticarial rash. 4 patients $(9.09 \%)$ showed neurosensory deafness since childhood. 3 patients $(6.8 \%)$ presented ocular involvement in form of conjunctivitis and/or uveitis. In 3 cases $(6.8 \%)$, antilL-1 (anakinra) or anti-TNF were used.

Conclusion: Cryopirinopathies are autoinflammatory diseases that occur mainly in the pediatric age, but there are also cases in adulthood. We must consider its diagnosis in those cases with periodic fever, arthralgia or arthritis, sensorineural deafness, APR elevation and urticarial rash. The genetic diagnosis will help us to confirm the diagnosis and avoid a delay in it.

Disclosure of Interests: None declared

DOI: 10.1136/annrheumdis-2019-eular.6805

\title{
FRI0611 ACUTE AND LONG-TERM EFFICACY OF IL-1 INHIBITOR ANAKINRA IN IDIOPATHIC AND SECONDARY PERICARDITIS IN PATIENTS REFRACTORY OR INTOLERANT TO CONVENTIONAL THERAPY
}

Muhammad Hamza Saad Shaukat ${ }^{1}$, Sukhraj Singh ${ }^{1}$, Kathryn Davis ${ }^{2}$, Mikhail Torosoff ${ }^{3}$, Ruben Peredo-Wende ${ }^{4} .{ }^{1}$ Albany Medical Center, Internal Medicine, Albany, NY, United States of America; ${ }^{2}$ Albany Medical College, Albany NY, United States of America; ${ }^{3}$ Albany Medical Center, Cardiology, Albany, NY, United States of America; ${ }^{4}$ Albany Medical Center, Rheumatology, Albany, United States of America

Background: In contrast to its use in idiopathic recurrent pericarditis (IRP), efficacy and optimal treatment duration of Anakinra are unclear in pericarditis secondary to known autoimmune or inflammatory conditions.

Objectives: a) Assess the efficacy of Anakinra in secondary and idiopathic pericarditis, refractory or intolerant to conventional therapy (CT; colchicine and corticosteroid [CS])

b) Study the effect of extended Anakinra continuation on post-treatment relapse risk.

Methods: Retrospective chart review of 12 adults hospitalised between January 2016 and October 2018 treated with Anakinra 100mg SQ daily for pericarditis refractory or intolerant to $\mathrm{CT}(\mathrm{CT}+\mathrm{A}$ group), and 22 consecutive hospitalised patients treated with $\mathrm{CT}$ only (CT group).

Results: Diagnosed autoimmune or inflammatory condition was present in $83.3 \%(10 / 12)$ of $C T+A$ and $40.9 \%(9 / 22)$ of $C T$ group $(p=0.07$, Table 1$)$ The most common etiologies in $\mathrm{CT}+\mathrm{A}$ group were RA and Undifferentiated Connective Tissue/Overlap Disease (Figure 1).

Despite CT-intolerance or resistance (Figure 2), all CT+A (12/12) patients had symptom relief at discharge vs. $72.7 \%(16 / 22)$ in $C T$ group $(p=0.04)$. There was a strong trend in $\mathrm{CT}+\mathrm{A}$ compared to $\mathrm{CT}$ group towards shorter time to symptom improvement $(1.75+/-1.29$ vs. $3.55+/-3.06$ days $\mathrm{p}=0.06)$ and symptom relief $(3.75+/-1.87$ vs $5.63+/-3.28$ days; $\mathrm{p}=0.08)$. CS were tapered successfully in $71.4 \%(5 / 7)$ vs. $66.7 \%(6 / 9)$ of patients in the $C T+A$ and CT groups $(p=0.56)$. Anakinra was associated with a longer mean length of stay $(13.0+/-16.2$ vs. $8.68+/-5.07$ days; $p=0.25)$.

No recurrence during treatment was noted in $C T+A$ group (0/12) vs. $40.9 \%(9 / 22)$ in CT group $(p=0.009)$. There was a trend towards reduced post-treatment relapse risk in CT+A group $[16.7 \%(2 / 12)$ vs. $41.7 \%(5 /$ 12), $\mathrm{p}=0.18]$. In relapsed patients, there was no significant difference in time to relapse $(1.75+/-0.34$ vs $1.72+/-0.71$ months, $\mathrm{p}=0.93)$. Within $C T+A$ group, extended Anakinra use in 6 patients (mean: 5.58+/-6.5 months) did not reduce post-treatment relapse risk $[16.7 \%(1 / 6)$ vs $16.7 \%(1 / 6)$ $p>0.9]$. Adverse effects were infrequent; elevated transaminases $(n=1)$ and local injection-site reaction $(n=1)$. Neither required permanent Anakinra discontinuation.

Table 1. Baseline Characteristics

\begin{tabular}{|c|c|c|c|}
\hline & $\begin{array}{c}\text { Anakinra plus } \\
\text { conventional therapy } \\
\%(n=12)\end{array}$ & $\begin{array}{l}\text { Conventional } \\
\text { therapy only } \\
\%(n=22)\end{array}$ & $\begin{array}{c}\mathrm{p}- \\
\text { value }\end{array}$ \\
\hline Age (years +/- SD) & $60.3+/-17.3$ & $56.8+/-17.8$ & 0.590 \\
\hline Female & $66.7(8)$ & $50(11)$ & 0.349 \\
\hline $\begin{array}{l}\text { Diagnosed autoimmune or } \\
\text { inflammatory condition }\end{array}$ & $83.3(10)$ & $40.9(9)$ & 0.074 \\
\hline $\begin{array}{l}\text { Recurrent pericarditis (>1 } \\
\text { episode) }\end{array}$ & $33.3(4)$ & $27.3(6)$ & 0.710 \\
\hline $\begin{array}{l}\text { Time since initial episode (months } \\
+/ \text {-SD) }\end{array}$ & $49.0+/-79.9$ & $9.21+/-11.7$ & 0.441 \\
\hline Prior NSAID use & $25.0(3)$ & $18.2(4)$ & 0.638 \\
\hline Prior Colchicine use & $25.0(3)$ & $9.09(2)$ & 0.210 \\
\hline Prior CS use & $50.0(6)$ & $18.2(4)$ & 0.051 \\
\hline Pericardial effusion on ECHO & $75.0(9)$ & $81.8(18)$ & 0.638 \\
\hline \multicolumn{4}{|l|}{ Treatment during hospitalisation } \\
\hline NSAID & $50.0(6)$ & $54.5(12)$ & 0.799 \\
\hline Colchicine & $58.3(7)$ & $63.6(14)$ & 0.761 \\
\hline CS & $58.3(7)$ & $40.9(9)$ & 0.331 \\
\hline \multicolumn{4}{|l|}{ Risk factors for pericarditis } \\
\hline $\begin{array}{l}\mathrm{PCl} \text {, cardiac surgery or chest } \\
\text { trauma }\end{array}$ & $16.7(2)$ & $45.5(10)$ & 0.093 \\
\hline Chest radiation & $8.33(1)$ & $4.55(1)$ & 0.653 \\
\hline
\end{tabular}

